\title{
Redesigned antibiotic combats drug-resistant tuberculosis
}

The emergence of tuberculosis (TB) infections that are resistant to multiple existing drugs is an increasingly important public health challenge. Reporting in Nature Medicine, Lee and colleagues now describe the generation of a novel class of anti-TB agents, called spectinamides, that show activity against multidrug-resistant TB.

From the 1940 s to 1960 s, numerous antibiotics were identified by screening soil-derived streptomycetes to identify natural products with antimicrobial properties. Although resistance to those natural products that were used clinically inevitably emerged, the development of semi-synthetic derivatives often allowed antibiotic activity to be regained. Eventually, however, this strategy - of iterative modification of a particular class — fails, and so there is an ongoing need to identify and optimize novel classes of antibiotics.

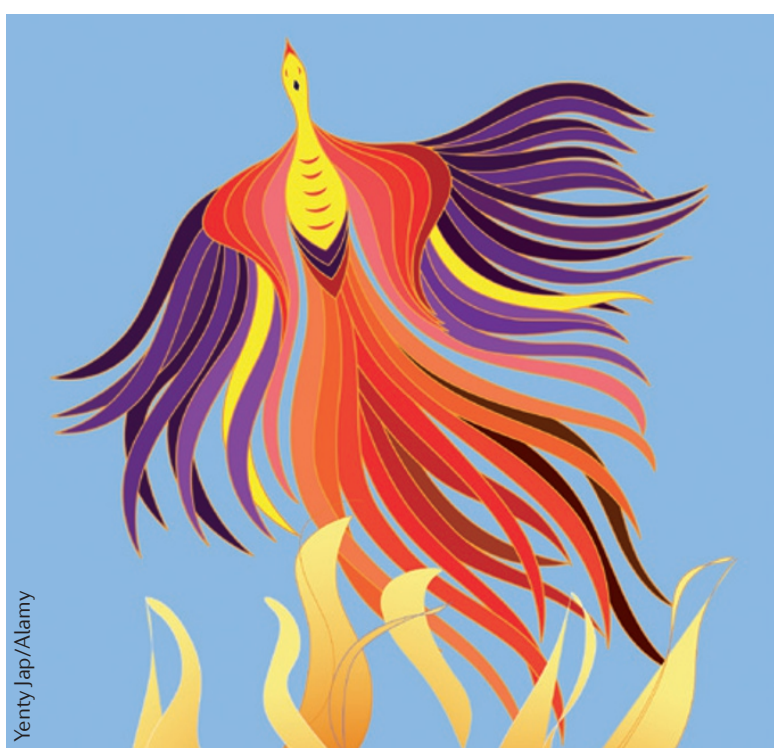

Growing knowledge of the structural basis for the activity of antibiotics is providing new opportunities to re-evaluate known antibiotic classes that may have previously been overlooked owing to issues such as lack of potency. In their study, the authors investigated spectinomycin, an antibiotic in a distinct structural class whose use so far has been limited to second-line treatment of gonorrhoea; structures of the antibiotic bound to its target - the $16 \mathrm{~S}$ bacterial ribosome subunit - have recently become available.

Spectinomycin itself has limited antimycobacterial activity, probably in part owing to poor cellular uptake and/or active expulsion from the bacteria. Indeed, a previous study found that genetic deletion of Rv1258c, a mycobacterial efflux pump, increases the anti-TB activity of spectinomycin. Using information obtained from the crystal structure of spectinomycin bound to the ribosome, and structural information obtained from antibiotics that avoid efflux pumps, spectinomycin derivatives were generated that were optimized for activity against TB. Further structural refinement, guided by molecular dynamic simulations of the drug bound to the ribosome, led to the identification of three additional promising spectinamides.

These spectinamides showed high activity against multidrug-resistant and extensively drug-resistant strains of Mycobacterium tuberculosis in in vitro assays and had no crossresistance with other antibiotics that inhibit protein synthesis and are used to treat TB, such as streptomycin. Deletion of Rv1258c did not affect the activity of the spectinamides, which suggests that, unlike spectinomycin, these compounds are not expelled by this pump.

Finally, in in vivo mouse models of both acute and chronic TB infection, spectinamides (injected subcutaneously at a dose of $200 \mathrm{mg}$ per $\mathrm{kg}$ twice daily or by direct intratracheal administration three times a week) were as effective at reducing the bacterial lung burden as streptomycin and/or rifampicin, which are currently used in TB therapy.

With regard to the safety profile of spectinamides, other in vitro experiments showed that spectinamides did not inhibit mitochondrial translation in mammalian cell lines, unlike other antibiotics that inhibit protein synthesis and therefore cause mitochondrial toxicity. They also do not inhibit the potassium channel ERG (which is linked to cardiotoxicity) or cytochrome P450 enzymes (thereby reducing the risk of drug-drug interactions). Given this, as well as their good pharmacokinetic properties, spectinamides might become a promising drug class for drugresistant $\mathrm{TB}$, even though their lack of oral bioavailability is an important challenge. Furthermore, the study highlights the potential for new structural knowledge to reinvigorate the investigation of semi-synthetic derivatives of other antibiotics to combat antibiotic resistance.

Megan Cully

ORIGINAL RESEARCH PAPER Lee, R. E. et al. Spectinamides: a new class of semisynthetic antituberculosis agents that overcome native drug efflux. Nature Med. 20, 152-158 (2014) FURTHER READING Zumla, A., Nahid, P. \& Cole, S. T. Advances in the development of new tuberculosis drugs and treatment regimens. Nature Rev. Drug Discov. 12, 388-404 (2013) 